\title{
Catadioptric camera model with conic mirror
}

\author{
G. López-Nicolás \\ gonlopez@unizar.es \\ C. Sagüés \\ csagues@unizar.es
}

\begin{abstract}
Catadioptric systems consist of the combination of lenses and mirrors. From them, central panoramic systems stand out because they provide a unique effective viewpoint, leading to the well-known unifying theory for central catadioptric systems. This paper considers catadioptric systems consisting of a conic mirror and a projective camera. Although a system with conic mirror does not possess a single projection point, it has some advantages as the cone is a very simple shape to produce, it has higher resolution in the peripheral, and adds less optical distortion to the images. The contributions of this work are the model of this non-central system by means of projective mappings from a torus to a plane, and the definition of the conic fundamental matrix with a role similar to that of perspective cameras. Additionally, a procedure to compute the relative motion between two views from the conic fundamental matrix is presented.
\end{abstract}

\section{Introduction}

Vision systems stand out from other types of sensors because they provide very rich information and because of their versatility and low cost. For the last years, the use of omnidirectional cameras is growing because of their effectiveness due to the panoramic view from a single image. A main class of cameras are the catadioptric systems, consisting of the combination of lenses and mirrors. Single viewpoint is a desirable property of a camera system, and the complete class of central catadioptric sensors with one mirror and lens are treated in [1]. A unifying theory for all central catadioptric systems was proposed in [6] and extended in [2]. In these works, the image formation model is developed by defining the well-known unified sphere model. Although having a single viewpoint is a desirable requirement in design, other features may be considered depending on the application. Usual central catadioptric cameras are built combining a hyperbolic mirror with a perspective camera or a parabolic mirror with a orthographic camera.

This paper considers catadioptric systems using a conic mirror and a projective camera. Some of the advantages compared with usual catadioptric systems are that the cone is a very simple shape to produce, it has higher resolution in the peripheral and adds less optical distortion to the images [11]. Another advantage compared with paracatadioptric systems is that a perspective camera is used instead of an expensive and complex orthographic camera. The use of conic mirror results in a non-central camera system. When the viewpoint of the perspective camera coincides with the vertex of the conic mirror, a central camera system is obtained [1]. This particular case has been studied in [11] showing its feasibility but reducing 
the configuration possibilities. Thus, despite non-centrality, the versatility of the conic mirror setup is a desirable property as shown for example in the single mirror stereo arrangement proposed in [3]. The field of view of the conic mirror system is smaller but more flexible. In fact, the smaller vertical field of view can be seen as an advantage because it provides higher angular resolution with the same number of pixels. For example, the camera is out of its field of view and it is not projected in the image plane (so part of the image is not wasted imaging the sensor itself) [12]. As said, the conic mirror and camera system is non-central, and the contribution of this work is the projection model for this system. This is achieved by exploting the concept of unitary sphere model to the unitary torus model and taking into account that the viewpoint of this system lies on a circular locus.

The estimation problem of the epipolar geometry using omnidirectional vision has been studied for central cameras [16], [7] or approximately central cameras [14]. Multi-view geometry is investigated in [15] considering a highly general imaging model using Plücker coordinates for central or non-central camera types. The epipolar geometry has been investigated for linear pushbroom cameras [9], for crossed-slits projection [4], and for circular panoramas [13]. In this work, the epipolar geometry of the catadioptric non-central camera system considered is tackled, and the conic fundamental matrix is defined with a role similar to the fundamental matrix of perspective cameras. The procedure to estimate the conic fundamental matrix from point correspondences is outlined. As application, camera motion between two conic views is obtained from this fundamental matrix.

The paper is organized as follows. Section 2 presents the camera model with a conic mirror. The conic fundamental matrix is derived in Section 3. Section 4 proposes the method to compute the relative motion between two views from the conic fundamental matrix. Simulations are provided in Section 5 to illustrate the proposal.

\section{Conic mirror imaging}

In this section we describe the geometry of the imaging system and define the catadioptric camera model using the conic mirror. The different parameters and coordinate systems involved are depicted in Fig. 1. The vertex of the conic mirror is $\mathbf{O}_{\mathbf{m}}$, and $\mathbf{O}_{\mathbf{c}}$ is the centre of the projective camera. The conic mirror and the camera are vertically aligned with respect the symmetry axis. The angle at the vertex of the cone is $2 \tau$ and the focal length of the camera is $f$. The distance from the mirror vertex to the camera centre is $f_{m}$. The origin of the coordinate system is the vertex of the cone with the $z$-axis aligned with the camera optical axis. It is known that the viewpoint of this camera system lies on a circular locus of radius $f_{x}$ vertically translated $f_{z}$ with respect to the vertex of the cone [1], where

$$
f_{x}=f_{m} \sin (2 \tau), \quad \text { and } \quad f_{z}=f_{m} \cos (2 \tau) .
$$

The parameter $\alpha$ is also defined as $(\alpha+\tau)=\pi / 2$.

\subsection{Point projection through the conic mirror}

Let us consider a world point $\mathbf{p}$ with coordinates $(X, Y, Z)^{T}$ in a general reference system with origin $\mathbf{O}_{\mathbf{m}}$ and $z$-axis aligned with the camera optical axis. For the subsequent development, it is interesting to align the $x$-axis of the reference with the vertical projection of the world point. This can be done by rotating the reference $\varphi=\arctan 2(Y, X)$ around the $z$-axis in such 


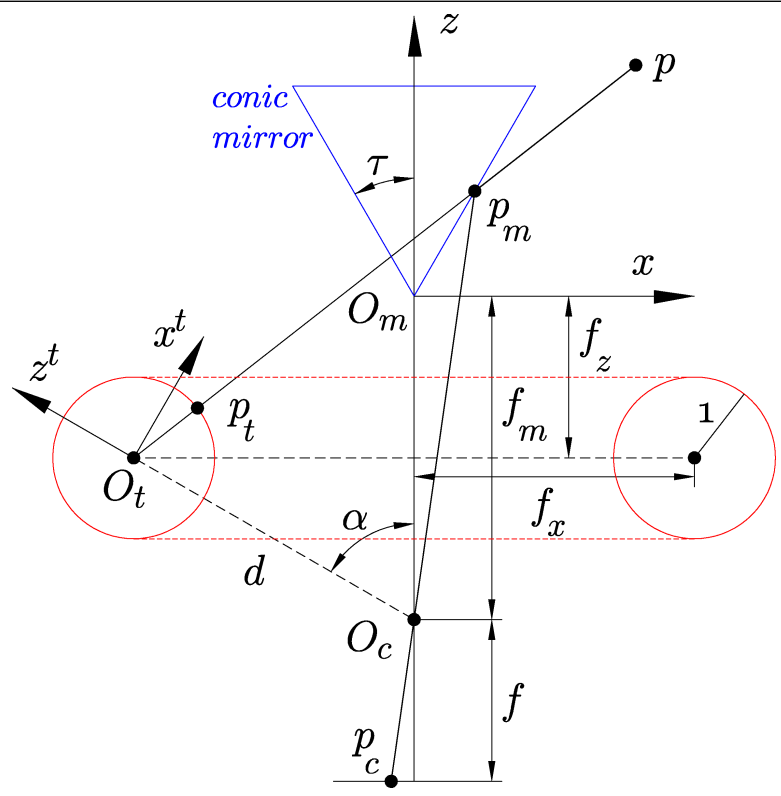

Figure 1: Geometry of the conic mirror and camera system. A world point $\mathbf{p}$ is projected in the image plane $\mathbf{p}_{\mathbf{c}}$ and the unitary torus $\mathbf{p}_{\mathbf{t}}$. The coordinate systems and geometric parameters are described in the text.

a way the new coordinates $\left(p_{x}, p_{y}, p_{z}\right)$ of $\mathbf{p}$ are obtained as

$$
p_{x}=\sqrt{X^{2}+Y^{2}}, \quad p_{y}=0, \quad \text { and } p_{z}=Z,
$$

This step can be performed with each world point in order to obtain its particular coordinate system with origin $\mathbf{O}_{\mathbf{m}}$ as depicted in Fig. 1 . Notice that, in this reference system, $p_{x}$ is the radial coordinate of the world point. Now, the center of projection of a particular world point is denoted $\mathbf{O}_{\mathbf{t}}$. Thus, the point $\mathbf{p}$ is projected through $\mathbf{O}_{\mathbf{t}}$ intersecting the mirror surface in $\mathbf{p}_{\mathbf{m}}$. The equation of the ray from $\mathbf{p}$ to $\mathbf{O}_{\mathbf{t}}$ is given by

$$
\frac{z-p_{z}}{x-p_{x}}-\frac{f_{z}+p_{z}}{f_{x}+p_{x}}=0
$$

On the other hand, the equation of the mirror surface is

$$
z \pm x / \tan \tau=0 \text {. }
$$

The coordinates $\left(x_{m}, z_{m}\right)$ of the point $\mathbf{p}_{\mathbf{m}}$ are the solution of (3) and (4). Next, the mirror point $\mathbf{p}_{\mathbf{m}}$ is projected through the optical centre of the camera $\mathbf{O}_{\mathbf{c}}$. The equation of the ray is

$$
\left(z_{m}+z\right) /\left(x_{m}-x\right)-\left(z_{m}+f_{m}\right) / x_{m}=0 .
$$

Finally, the intersection of this ray with the image plane yields

$$
x_{c}=x_{m}-x_{m}\left(f+f_{m}+z_{m}\right) /\left(f_{m}+z_{m}\right),
$$

which is a radial coordinate that determines, together with $\varphi$, the image projection $\mathbf{p}_{\mathbf{c}}$. 


\subsection{The unitary torus model}

The unitary torus model is inspired on the idea of the unified sphere model for central cameras presented in [6], [2]. In central cameras, a section of the unitary sphere along the symmetry axis gives a unitary circle. In our case, the revolution of the unitary circle in each viewpoint across the circular locus yields a torus. Firstly, we define the coordinate system $\left(x^{t}, z^{t}\right)$ with origin $\mathbf{O}_{\mathbf{t}}$ and $z$-axis aligned with the segment $\overline{\mathbf{O}_{\mathbf{t}} \mathbf{O}_{\mathbf{c}}}$ as shown in Fig. 1 . In the following, the superindex $t$ denotes that the value is referred to the $\mathbf{O}_{\mathbf{t}}$ coordinate system. The distance $d$ can also be computed using

$$
d=\overline{\mathbf{O}_{\mathbf{t}} \mathbf{O}_{\mathbf{c}}}=\sqrt{f_{x}^{2}+\left(f_{m}-f_{z}\right)^{2}}=2 f_{m} \sin \tau .
$$

The general equation of the torus particularized to the unitary torus is defined as

$$
\left(f_{x}-\sqrt{x^{2}+y^{2}}\right)^{2}+\left(z-f_{z}\right)^{2}=1,
$$

where the centre of the tube is the circle of radius $f_{x}$ (i.e. the circular locus on which the viewpoint of the camera system lies) and the radius of the tube is the unit. The section of the torus is represented in Fig. 1 with two symmetric circles. The point on the mirror $\mathbf{p}_{\mathbf{m}}$ in the coordinate system with origin in $\mathbf{O}_{\mathbf{t}}$ is given by $x_{m}^{t}=f_{x}^{t}+z_{m} / \cos \tau$ and $z_{m}^{t}=f_{z}^{t}$, where $f_{x}^{t}=f_{x} \cos \alpha+f_{z} \sin \alpha$ and $f_{z}^{t}=-f_{x} \sin \alpha+f_{z} \cos \alpha$. The point on the unitary torus can be obtained normalizing the point coordinates and projecting to the image plane yielding $x_{c}^{t}=f x_{m}^{t} / f_{z}^{t}$, which is the projection of the world point up to a rotation of the reference system. Finally, the image point can be obtained as

$$
x_{c}=\frac{x_{c}^{t}+f \tan \alpha}{1-\left(x_{c}^{t} / f\right) \tan \alpha},
$$

Which gives an equivalent result as in (6). This procedure can be carried out inversely to reproject any image point to the unitary torus. Then, given the coordinates $x_{c}$ and $\varphi$ of a point in the image, it can be reprojected onto the torus following these steps:

1. Coordinate transformation: $x_{c}^{t}=\frac{x_{c}-f \tan \alpha}{1+\left(x_{c} / f\right) \tan \alpha}$

2. Reproject to the unitary torus obtaining the $\mathbf{p}_{\mathbf{t}}^{\mathbf{t}}$ with coordinates $\left(x_{t}^{t}, z_{t}^{t}\right)$ in the reference system at $\mathbf{O}_{\mathbf{t}}: x_{t}^{t}=\lambda x_{c}^{t} / f$, and $z_{t}^{t}=\lambda$ with $\lambda=1 / \sqrt{1+\left(x_{c}^{t} / f\right)^{2}}$

3. Coordinate transformation: $x_{t}=x_{t}^{t} \cos \alpha-z_{t}^{t} \sin \alpha$, and $z_{t}=x_{t}^{t} \sin \alpha+z_{t}^{t} \cos \alpha$

The result is a point $\mathbf{p}_{\mathbf{t}}$ projected onto the torus $\left(x_{t}, z_{t}, \varphi\right)$, playing a similar role as the point in the unitary sphere for central cameras. This is a unified representation with a model based on the unitary torus.

\section{The conic fundamental matrix}

The epipolar geometry represents the relative geometry between two views of a scene. The fundamental matrix is the algebraic representation of this epipolar geometry and it is used to formulate the epipolar constraint for image correspondences. It is independent of the scene structure and only depends on the relative configuration of the cameras and their intrinsic 
parameters [10]. In this section, a novel conic fundamental matrix is presented with a role similar to the fundamental matrix for perspective cameras.

Let us suppose the camera matrices of two views are $\mathbf{P}$ and $\mathbf{P}^{\prime}$ with the origin of the global reference at the second camera. Representing the points of the world and the points on the unitary torus by homogeneous coordinates, the projection can be represented as

$$
\left(\begin{array}{c}
x_{t} \\
y_{t} \\
1
\end{array}\right)=\mathbf{P}\left(\begin{array}{c}
X \\
Y \\
Z \\
1
\end{array}\right), \quad\left(\begin{array}{c}
x_{t}^{\prime} \\
y_{t}^{\prime} \\
1
\end{array}\right)=\mathbf{P}^{\prime}\left(\begin{array}{c}
X \\
Y \\
Z \\
1
\end{array}\right),
$$

where $(X, Y, Z, 1)^{T}$ is a world point and $\left\{\left(x_{t}, y_{t}, 1\right),\left(x_{t}^{\prime}, y_{t}^{\prime}, 1\right)\right\}$ are two correspondent points on the unitary torus. Notice that the points have been normalized by the third coordinate $\left(z_{t}, z_{t}^{\prime}\right)$. The camera matrices are represented by $3 \times 4$ matrices defined as

$$
\begin{aligned}
\mathbf{P} & =\left[\begin{array}{ll}
\mathbf{R}_{\mathbf{c}} & \mathbf{T}_{\mathbf{c}}
\end{array}\right]\left[\begin{array}{cc}
\mathbf{R} & \mathbf{T} \\
\mathbf{0} & 1
\end{array}\right]^{-1} \\
\mathbf{P}^{\prime} & =\left[\begin{array}{ll}
\mathbf{R}_{\mathbf{c}}^{\prime} & \mathbf{T}_{\mathbf{c}}^{\prime}
\end{array}\right]\left[\begin{array}{cc}
\mathbf{R}^{\prime} & \mathbf{T}^{\prime} \\
\mathbf{0} & 1
\end{array}\right]^{-1},
\end{aligned}
$$

The rotation and translation of the first camera with respect to the origin are given by

$$
\mathbf{R}=\left[\begin{array}{lll}
r_{11} & r_{12} & r_{13} \\
r_{21} & r_{22} & r_{23} \\
r_{31} & r_{32} & r_{33}
\end{array}\right], \mathbf{T}=\left(\begin{array}{c}
t_{x} \\
t_{y} \\
t_{z}
\end{array}\right)
$$

Without loss of generality we have assumed the second camera in the origin, and thus $\mathbf{R}^{\prime}=\mathbf{I}$ and $\mathbf{T}^{\prime}=\mathbf{0}$. Finally, the part related with the projection on the unitary torus is defined for both cameras as

$$
\mathbf{R}_{\mathbf{c}}=\left[\begin{array}{ccc}
\cos \varphi & \sin \varphi & 0 \\
-\sin \varphi & \cos \varphi & 0 \\
0 & 0 & 1
\end{array}\right], \mathbf{R}_{\mathbf{c}}^{\prime}=\left[\begin{array}{ccc}
\cos \varphi^{\prime} & \sin \varphi^{\prime} & 0 \\
-\sin \varphi^{\prime} & \cos \varphi^{\prime} & 0 \\
0 & 0 & 1
\end{array}\right], \mathbf{T}_{\mathbf{c}}=\mathbf{T}_{\mathbf{c}}^{\prime}=\left(\begin{array}{c}
f_{x} \\
0 \\
f_{z}
\end{array}\right)
$$

where $\varphi$ has been previously defined and $\varphi^{\prime}$ is analogous, but including the motion of the first camera. The projection equations (10) can be written in a different form as

$$
\left[\begin{array}{c|cc}
\mathbf{P} & x_{t} & 0 \\
& y_{t} & 0 \\
& 1 & 0 \\
\hline & 0 & x_{t}^{\prime} \\
\mathbf{P}^{\prime} & 0 & y_{t}^{\prime} \\
& 0 & 1
\end{array}\right] \quad\left(\begin{array}{c}
X \\
Y \\
Z \\
1 \\
-z_{t} \\
-z_{t}^{\prime}
\end{array}\right)=\mathbf{0} .
$$

The $6 \times 6$ previous matrix is denoted $\mathbf{A}$. The previous equation must hold for any point of the scene and therefore, $\operatorname{det}(\mathbf{A})=0$. Developing the determinant of $\mathbf{A}$ and rearranging terms, it can be proved that there exists a $5 \times 5$ matrix $\mathbf{F}$ that we call the conic fundamental matrix satisfying

$$
\left(\begin{array}{c}
\cos \varphi^{\prime} \\
\sin \varphi^{\prime} \\
x_{t}^{\prime} \cos \varphi^{\prime} \\
x_{t}^{\prime} \sin \varphi^{\prime} \\
1
\end{array}\right)^{T} \mathbf{F}\left(\begin{array}{c}
\cos \varphi \\
\sin \varphi \\
x_{t} \cos \varphi \\
x_{t} \sin \varphi \\
1
\end{array}\right)=0
$$


The entries of $\mathbf{F}$ only depend on the two camera matrices $\left(\mathbf{P}, \mathbf{P}^{\prime}\right)$. Notice also that the lifted coordinates of the image point correspondences on the unitary torus do not depend on the coordinates $\left(y_{t}, y_{t}^{\prime}\right)$. This is because of the axial symmetry of the catadioptric camera system and the selected coordinate reference system defined for the imaging model. Finally, the conic fundamental matrix is given by

$$
\mathbf{F}=\left[\begin{array}{ccccc}
0 & 0 & f_{13} & f_{14} & f_{15} \\
0 & 0 & f_{23} & f_{24} & f_{25} \\
f_{31} & f_{32} & f_{33} & f_{34} & f_{35} \\
f_{41} & f_{42} & f_{43} & f_{44} & f_{45} \\
f_{51} & f_{52} & f_{53} & f_{54} & f_{55}
\end{array}\right]
$$

where the conic fundamental matrix entries are defined as

$$
\begin{array}{lll}
f_{31}=-r_{21} f_{x} \quad f_{32}=r_{11} f_{x} & f_{33}=-r_{21} r_{33} f_{z}+r_{21}\left(t_{z}+f_{z}\right)+r_{31} r_{23} f_{z}-r_{31} t_{y} \\
f_{41}=-r_{22} f_{x} \quad f_{42}=r_{12} f_{x} & f_{34}=r_{11} r_{33} f_{z}-r_{11}\left(t_{z}+f_{z}\right)-r_{31} r_{13} f_{z}+r_{31} t_{x} \\
f_{51}=-r_{23} f_{x} \quad f_{52}=r_{13} f_{x} & f_{35}=-r_{11} r_{23} f_{z}-r_{21} t_{x}+r_{11} t_{y}+r_{21} r_{13} f_{z} \\
f_{13}=r_{21} r_{33} f_{x}-r_{31} r_{23} f_{x} & f_{43}=-r_{22} r_{33} f_{z}+r_{22}\left(t_{z}+f_{z}\right)+r_{32} r_{23} f_{z}-r_{32} t_{y} \\
f_{14}=-r_{11} r_{33} f_{x}+r_{31} r_{13} f_{x} & f_{44}=r_{12} r_{33} f_{z}-r_{12}\left(t_{z}+f_{z}\right)-r_{32} r_{13} f_{z}+r_{32} t_{x} \\
f_{15}=r_{11} r_{23} f_{x}-r_{21} r_{13} f_{x} & f_{45}=r_{22} r_{13} f_{z}-r_{22} t_{x}-r_{12} r_{23} f_{z}+r_{12} t_{y} \\
f_{23}=r_{22} r_{33} f_{x}-r_{32} r_{23} f_{x} & f_{53}=r_{23}\left(t_{z}+f_{z}\right)-r_{33} t_{y} \\
f_{24}=-r_{12} r_{33} f_{x}+r_{32} r_{13} f_{x} & f_{54}=-r_{13}\left(t_{z}+f_{z}\right)+r_{33} t_{x} \\
f_{25}=r_{12} r_{23} f_{x}-r_{22} r_{13} f_{x} & f_{55}=r_{13} t_{y}-r_{23} t_{x}
\end{array}
$$

\subsection{Estimation of $\mathbf{F}$ from point correspondences}

The conic fundamental matrix can be computed from a set of point correspondences, without knowledge of the relative camera positions, using the constraint (16) by solving a linear system of equations. The lifted coordinates are obtained from the normalized points of the unitary torus: $\left(x_{t}, y_{t}, 1\right)$ and $\left(x_{t}^{\prime}, y_{t}^{\prime}, 1\right)$. Each point correspondence gives an equation. Given that $\mathbf{F}$ is defined by 21 unknown entries, a set of 20 point correspondences allows to determine $\mathbf{F}$ up to a scale factor by solving a linear system of equations. In general, more than the minimum set of correspondences are available and the presence of image noise or mismatches is assumed. Then, a robust method for estimation like RANSAC [5] can be used.

\subsection{Epipolar curves}

The epipolar geometry for perspective cameras involves the epipoles, defined as the intersection point of the baseline with the image plane, and the epipolar lines, which are straight lines defined as the intersection of the plane containing the baseline with the image plane [10]. These geometric entities do not represent the corresponding conic mirror and camera system constraints. Let us consider the lifted coordinates of a point correspondence in two views satisfying the constraint (16). Fixing a point in the first view, all the possible matched points are obtained as

$$
\left(\begin{array}{l}
a \\
b \\
c \\
d \\
e
\end{array}\right)=\mathbf{F}\left(\begin{array}{c}
\cos \varphi \\
\sin \varphi \\
x_{t} \cos \varphi \\
x_{t} \sin \varphi \\
1
\end{array}\right)
$$


where $(a, b, c, d, e)^{T}$ determines the epipolar curve. The corresponding point in the second camera system lies on the epipolar curve, therefore

$$
\left(\begin{array}{c}
\cos \varphi^{\prime} \\
\sin \varphi^{\prime} \\
x_{t}^{\prime} \cos \varphi^{\prime} \\
x_{t}^{\prime} \sin \varphi^{\prime} \\
1
\end{array}\right)^{T}\left(\begin{array}{l}
a \\
b \\
c \\
d \\
e
\end{array}\right)=0
$$

This previous equation plays a similar role for the epipolar curve than the epipolar line of projective cameras.

\section{Extraction of motion from $\mathrm{F}$}

The relative motion between two projective cameras can be computed from the decomposition of the fundamental matrix as summarized in [10]. With the same goal, a procedure for the motion extraction given the conic fundamental matrix $\mathbf{F}$ (17) is presented. Firstly, the rotation matrix ( $\mathbf{R}=r_{i j}$ with $\left.i, j=1,2,3\right)$ between the two cameras is computed by retrieving the three rows of $\mathbf{R}$ separately. On the one hand, the first row of $\mathbf{R}$ can be obtained from the second column of $\mathbf{F}$ :

$$
\left(r_{11}, r_{12}, r_{13}\right)^{T}=\frac{-\left(f_{32}, f_{42} f_{52}\right)^{T}}{\left\|\left(f_{32}, f_{42}, f_{52}\right)\right\|} .
$$

On the other hand, the second row of $\mathbf{R}$ can be obtained from the first column of $\mathbf{F}$ :

$$
\left(r_{21}, r_{22}, r_{23}\right)^{T}=\frac{-\left(f_{31}, f_{41} f_{51}\right)^{T}}{\left\|\left(f_{31}, f_{41}, f_{51}\right)\right\|} .
$$

Finally, the third row of $\mathbf{R}$ can be computed solving a linear system derived from the first and second rows of $\mathbf{F}$ :

$$
\left[\begin{array}{rrrr}
-r_{23} & 0 & r_{21} & -f_{13} \\
0 & -r_{23} & r_{22} & -f_{23} \\
r_{13} & 0 & -r_{11} & -f_{14} \\
0 & r_{13} & -r_{12} & -f_{24}
\end{array}\right]\left(\begin{array}{c}
r_{31} \\
r_{32} \\
r_{33} \\
1
\end{array}\right)=\mathbf{0}
$$

After solving the system, the norm $\left\|\left(r_{31}, r_{32}, r_{33}\right)\right\|$ is used to normalize the resultant vector $\left(r_{31}, r_{32}, r_{33}\right)^{T}$. Alternatively, the third row of $\mathbf{R}$ can be obtained from the cross product of the other two rows. Notice that each row of $\mathbf{R}$ has been normalized to hold with the rotation matrix properties, i.e. that each row (or column) has unitary norm. Notice also that there are two solutions for the rotation depending on the sign factor of the fundamental matrix $(\mathbf{F},-\mathbf{F})$ leading to $(\mathbf{R},-\mathbf{R})$. The correct solution may be selected by testing point correspondences with both solutions.

Secondly, the translation vector $\mathbf{T}=\left(t_{x}, t_{y}, t_{z}\right)^{T}$ between the two cameras is computed solving a linear system which is defined by using the entries of the conic fundamental matrix 

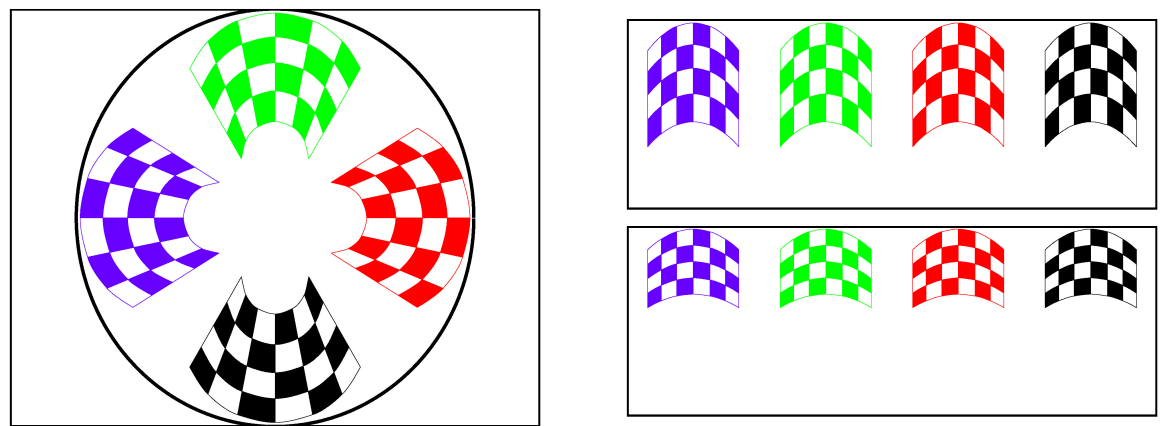

Figure 2: Example of an image acquired with the conic mirror and camera system (left). Unwarping by direct mapping from polar coordinates to Cartesian coordinates (right-top) and unwarping using the unitary torus model (right-below).

$f_{i j}$ with $i, j=3,4,5$. Denoting $t_{f z}=t_{z}+f_{z}$ we can write

$$
\left[\begin{array}{rrrr}
0 & -r_{31} & r_{21} & \left(r_{31} r_{23}-r_{21} r_{33}\right) f_{z}-f_{33} \\
0 & -r_{32} & r_{22} & \left(r_{32} r_{23}-r_{22} r_{33}\right) f_{z}-f_{43} \\
0 & -r_{33} & r_{23} & -f_{53} \\
r_{31} & 0 & -r_{11} & \left(r_{11} r_{33}-r_{31} r_{13}\right) f_{z}-f_{34} \\
r_{32} & 0 & -r_{12} & \left(r_{12} r_{33}-r_{32} r_{13}\right) f_{z}-f_{44} \\
r_{33} & 0 & -r_{13} & -f_{54} \\
-r_{21} & r_{11} & 0 & \left(r_{21} r_{13}-r_{11} r_{23}\right) f_{z}-f_{35} \\
-r_{22} & r_{12} & 0 & \left(r_{22} r_{13}-r_{12} r_{23}\right) f_{z}-f_{45} \\
-r_{23} & r_{13} & 0 & -f_{55}
\end{array}\right]\left(\begin{array}{c}
t_{x} \\
t_{y} \\
t_{f z} \\
1
\end{array}\right)=\mathbf{0}
$$

Solving the previous system, the translation is obtained up to a scale as $\mathbf{T}=\left(t_{x}, t_{y}, t_{f z}-f_{z}\right)^{T}$.

\section{Experiments}

In this section, we present simulations to illustrate the proposal. The camera system is defined with the configuration depicted in Fig. 1 using the following parameters: $\tau=\pi / 6$, $f_{m}=40 \mathrm{~mm}$ and $f=10 \mathrm{~mm}$. The size of the acquired images is $800 \times 600$ pixels with optical center $(400,300)$ pixels. The scene consists of four vertical planes with squared pattern. An example of an image acquired is shown in Fig. 2.

Some applications may require unwarping the omnidirectional images into panoramic images. Different methods to transform images acquired with hypercatadioptric systems into panoramic images were described in [8]. In the example of Fig. 2, a panoramic image is created from the omnidirectional image acquired with the conic mirror system by mapping the image pixels into a cylindrical plane. The direct mapping from polar coordinates to Cartesian coordinates is compared with the image unwarping using the unitary torus model proposed (Fig. 2, right). The results show that the distortions produced by the conic shape of the mirror are removed, and only remains the perspective distortion and the usual distortion of panoramic images.

In the next experiment, the camera system is translated and rotated arbitrarily along time with $t_{x}=2 \cos (t)+5, t_{y}=4 \sin (2 t)-5, t_{z}=1$ for the translation and $r_{x}=10 \sin (t), r_{y}=-8$, $r_{z}=10-2 t$ for the rotation with respect to each Cartesian axis. The point correspondences 

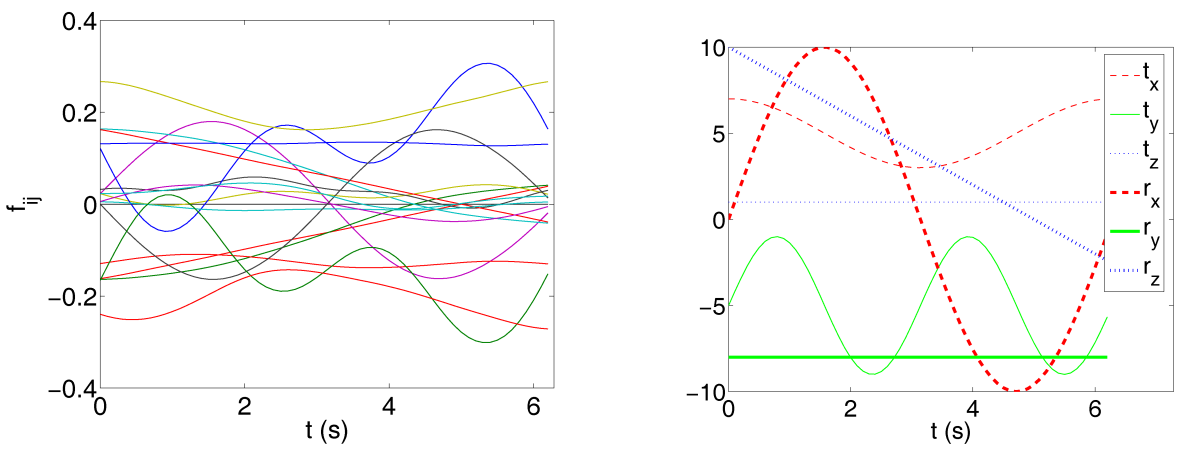

Figure 3: Evolution of the conic fundamental matrix entries $f_{i j}$ as a result of the camera motion (left). Extraction of the relative camera motion ( $m$ and deg) from the conic $\mathbf{F}$ (right).

between the image with the camera system at the origin and the images acquired by he camera system translated and rotated are used to compute the conic fundamental matrix. The evolution of the normalized conic fundamental matrix entries is shown (Fig. 3, left). The relative motion is also computed from the conic fundamental matrix coinciding with the motion defined (Fig. 3, right).

\section{Conclusion}

A novel model for catadioptric camera systems with conic mirrors has been presented. The contributions are the conic mirror imaging model based on the unitary torus model, the conic fundamental matrix definition and the procedure to compute relative motion between two conic views. The advantages of using conic mirrors in the acquisition system has been previously acknowledged. The interest of the proposal is that the definition of the model allows to use the non-central camera system with conic mirror in a simple way.

\section{Acknowledgment}

This work was supported by projects DPI2009-08126 and DPI2009-14664-C02-01.

\section{References}

[1] S. Baker and S. K. Nayar. A theory of single-viewpoint catadioptric image formation. International Journal of Computer Vision, 35(2):175-196, 1999.

[2] J. P. Barreto and H. Araujo. Issues on the geometry of central catadioptric image formation. IEEE Conference on Computer Vision and Pattern Recognition, 2:422-427, 2001.

[3] C. Burbridge, U. Nehmzow, and J. Condell. Omnidirectional projections with a cone mirror and single mirror stereo. In The 8th Workshop on Omnidirectional Vision, Camera Networks and Non-classical Cameras (OMNIVIS), 2008. 
[4] D. Feldman, T. Pajdla, and D. Weinshall. On the epipolar geometry of the crossed-slits projection. In Proceedings of International Conference on Computer Vision, pages 988-995, 2003.

[5] M. A. Fischler and R. C. Bolles. Random sample consensus: a paradigm for model fitting with applications to image analysis and automated cartography. Commun. ACM, 24(6):381-395, 1981.

[6] C. Geyer and K. Daniilidis. A unifying theory for central panoramic systems and practical applications. In Proceedings of the 6th European Conference on Computer Vision, ECCV-Part II, pages 445-461. Springer-Verlag, 2000.

[7] C. Geyer and K. Daniilidis. Mirrors in motion: epipolar geometry and motion estimation. IEEE International Conference on Computer Vision, 2:766-773, Oct 2003.

[8] V. Grassi and J. Okamoto. Development of an omnidirectional vision system. Journal of the Brazilian Society of Mechanical Sciences and Engineering, 28:58-68, 2006.

[9] R. Gupta and R. I. Hartley. Linear pushbroom cameras. IEEE Transactions on Pattern Analysis and Machine Intelligence, 19(9):963-975, 1997.

[10] R. I. Hartley and A. Zisserman. Multiple View Geometry in Computer Vision. Cambridge University Press, second edition, 2004.

[11] S.-S Lin and R. Bajcsy. True single view point cone mirror omni-directional catadioptric system. In IEEE International Conference on Computer Vision, volume 2, pages 102-107, 2001.

[12] S.-S. Lin and R. Bajcsy. High resolution catadioptric omni-directional stereo sensor for robot vision. In IEEE International Conference on Robotics and Automation, pages 1694-1699, 2003.

[13] M. Menem and T. Pajdla. Constraints on perspective images and circular panoramas. In British Machine Vision Conference, September 2004.

[14] B. Micusik and T. Pajdla. Structure from motion with wide circular field of view cameras. IEEE Transactions on Pattern Analysis and Machine Intelligence, 28(7):11351149 , July 2006.

[15] P. Sturm. Multi-view geometry for general camera models. In Proceedings of the IEEE Conference on Computer Vision and Pattern Recognition, volume 1, pages 206-212, San Diego, California, USA, June 2005.

[16] T. Svoboda and T. Pajdla. Epipolar geometry for central catadioptric cameras. International Journal of Computer Vision, 49(1):23-37, 2002. 